\title{
Development Trends for China's Instrumentation Engineering Science and Technology to 2035
}

\author{
Wang Xue ${ }^{1}$, Zhang $\mathrm{Li}^{2}$ \\ 1. Tsinghua University, Beijing 100084, China \\ 2. China Instrument and Control Society, Beijing 100088, China
}

\begin{abstract}
Instrumentation engineering science and technology is the fundamental technology for several applications including intelligent manufacturing, scientific research, environmental monitoring, medical health, and national defense. Instrumentation engineering science and technology is an important support toward achieving a national innovation strategy that determines the development level of strategic emerging industries, such as the emerging information industry, high-end equipment-manufacturing industry, new energy industry, and new materials industry. The present study involves analyzing development trends in instrumentation until the year 2035. Given the requirements of digital, intelligent, and networked instruments, key technologies and future trends are depicted for the following three main aspects: intelligent instruments, medical instruments, and micro-electromechanical systems (MEMS). Development strategies and suggestions for instrumentation engineering science and technology in 2035 are also presented.
\end{abstract}

Keywords: instrumentation; intelligent instrumentation; medical instrument; MEMS; trend analysis

\section{Introduction}

Instrumentation engineering science and technology is essential for supporting technology and equipment for intelligent manufacturing, scientific research, environmental monitoring, medical health, and national defense construction, among others. A wellknown scientist and academician, Wang Daheng, once figuratively described it as the "multiplier" of industrial production, "pioneer" of scientific research, "fighting strength" of military affairs, and "materialized judge" of modern social activities [1].

A crucial support for achieving the national innovation-driven strategy is the development level of instrumentation engineering science and technology that determines the development of the emerging information industry, high-end equipment manufacturing industry, new energy and new materials, and other strategic emerging industries [2]. The development of digital, intelligent, and novel networked instrumentation serves as an important means to achieve intelligent sensing and decision control of measurement information, and it is an important aspect of intelligent manufacturing of equipment. The development level of instrumentation engineering science and technology is an important indicator of the innovation level and manufacturing capacity of a country [3-5].

Instrumentation involves a wide range of technical areas and is highly sensitive to new technologies. It is an area in modern industry with updated products and with extremely rapid application and development of new technologies. The research and development of its key technologies involve comprehensive cross-application of mathematics, mechanics, materials science, industry, electronics, informatics, cybernetics, artificial intelligence, and other disciplines and several boundary sciences.

The design, development, and manufacturing of instrumentation products involve sensitive mechanisms, sensitive materials, technology, process equipment, testing technology, and other basic research; thus, it is difficult to tackle key problems. The development of its industry not only involves the whole level of

Received date: 15 December 2016; revised date: 27 December 2016

Corresponding author: Wang Xue, Tsinghua University, Professor. Major research fields include precision measurement, intelligent sensing technology, and measurement and control technology of Internet of Things. E-mail: wangxue@mail.tsinghua.edu.cn

Funding program: CAE Advisory Project “Research on China’s Engineering Science and Technology Development Strategy 2035” (2015-ZD-14)

Chinese version: Strategic Study of CAE 2017, 19 (1): 103-107

Cited item: Wang Xue et al. Development Trends for China's Instrumentation Engineering Science and Technology to 2035. Strategic Study of CAE, https://doi. org/10.15302/J-SSCAE-2017.01.015 
technology, but is also closely related to engineering application technology.

Instrumentation is widely applied in all aspects of industry, agriculture, national defense, scientific research, and human life. Special technical research will be performed to satisfy different application needs and develop personalized solutions for users.

The above characteristics in the fields of instrumentation determine the high requirements and immense demand for the development of instrumentation engineering science technology for specialized and interdisciplinary talents.

In summary, it is very significant for industry development and government decision-making to establish the development goals of China's instrumentation in engineering science and technology for 2035, put forward a number of key tasks that are of great demand and consistent with the major development trends, emphasize the key points and new highlights, and further study the path of research and the development in depth.

The research front of instrumentation engineering science and technology mainly focuses on high-sensitivity "sources" and "detectors," new sensing technology, and interconnection technology of intelligent sensing systems, and generic technology of micro-electromechanical systems (MEMS). In applied technology, breakthroughs in the nondestructive, rapid, and online detection technology are necessary for tracing the quantitative detection technology and fully integrating the gene detection system. Mutual integration among sensor and signal processing, control circuits, and micro-actuators based on the MEMS technology will be achieved to form a micro-system with certain complete functions that correspond to the research and input focus in the industry. Its scale effect and the prospects of expanding from its applications will revolutionize personal consumption, transportation, industrial control, military affairs and national defense, and several other areas $[6,7]$.

In this study, the Delphi method is applied to technology forecast and studies to determine the key and generic technologies that shall be used to establish the development trends of instrumentation engineering science and technology in 2035 in China.

\section{Development goals}

The development of China's instrumentation engineering technology will be achieved by tracing information technology, nanotechnology, biotechnology, and other high-tech technology, researching current and emerging technology, and making breakthroughs in the development of key instrumentation to effectively narrow the gap with foreign countries. Plans include the development of the instrumentation industry-related materials, components, and equipment in a systematic and well-organized manner and to build an industrial structure in a wide range of fields as well as a relatively reasonable structure to create the instrumentation product system of the international advanced level. High-quality and high-standard development principles will be adhered to and the long-term constraints of reliability and stability issues on the development of China's instrumentation will be broken to enhance the capacity of engineering and industrialization of instrumentation in China.

The strategic goal of 2025 involves (i) achieving breakthroughs in acquisition and sensing technologies of physical quantities and extending the applied range of instrumentation to perform environmental pollution monitoring, nuclear radiation safety monitoring, material composition analysis, biochemical analysis, new energy and green energy development, and address other problems; (ii) using research achievements in quantum mechanics, nanoelectronics, terahertz ( $\mathrm{THz}$ ) electromagnetics, measurement technology of Internet of Things, artificial intelligent information processing, and other emerging disciplines to address bottleneck problems that restrict the development of instrumentation technology, and achieve breakthroughs in key technologies and engineering applications; and (iii) providing highend instruments and equipment for scientific research, energy development, and healthy life. The strategic goals to be achieved by 2035 involve, essentially, (i) achieving localization of highend instrumentation required for the China's development and improving the technical system of high-end instruments; (ii) building a remote measurement and fault diagnosis platform with "the network as the center" and an embedded measurement platform; and (iii) performing breakthroughs in instrumentation failure prediction and health management technology to achieve zero failure, self-repair, free maintenance, and other intelligent functions of the instrumentation. The overall level of China's instrumentation has reached an advanced level, and this is also applicable to product quality and reliability $[8,9]$.

\section{Development trends}

In the year 2035, attractive application prospects in areas such as quantum computer and quantum communication, stem cell and regenerative medicine, synthetic biology and artificial chloroplast, nanotechnology and quantum dot technology, and graphene materials in conjunction with the exploitation of new materials, life sciences, space utilization, ocean development, new energy, and other fields, would have increased the need for instrument precision [3].

Advanced manufacturing is developed in the direction of integration of structure and function and integration of materials and device. Extreme manufacturing technology is rapidly being pushed to the maximum (such as in aircraft carriers and ultralarge-scale integration) and to the minimum (such as in microand nano-chips) directions. The intelligent manufacturing mode of man-machine integration and 4D printing technology from the combination of smart materials and $3 \mathrm{D}$ printing promotes industries to transition from mass centralized production to customized distributed production, thereby leading the "materialization of the digital world" and the "intelligentization of the material 
world". Instrumentation is the basic support for industrial production that definitely satisfies the needs of new industries to generate new measurement methods [10].

\subsection{Field of intelligent instrumentation}

This involves, (i) developing sensor fusion sensing technology to monitor multiple parameters such as vibration, temperature, pressure, noise, strain, and images; (ii) developing multi-source self-powered micro-power continuous sensor to fundamentally solve the power supply issues in the front end of measurement; and (iii) making complete use of a wireless communication system and network platform to achieve a weather-independent and full-space sensing and monitoring, and to achieve sensing that is free from space and environmental restrictions [11].

This is combined with the artificial intelligence method to achieve breakthroughs in a number of key ontology detection technologies, improve the intelligent level of control of instrumentation ontology, build self-diagnosis and other advanced intelligent functions, and construct the gathering capacity of field data of ontology for automatic tracking in order to build a full life-cycle quality tracking system of the instrumentation [12].

Additionally, this involves building a sensor measurement architecture based on the Internet of Things from the most powerful applications to bottom sensing to construct the end-to-end big data transmission link and the big data network. It also involves breakthroughs in the local operation status of standalone instrumentation and building a seamless access to fieldbus or distributed control system (DCS) network to achieve access to communication network integration to instrumentation technology. Furthermore, this includes enriching instrumentation data information, extending the diversity of the data source with the exception of extending the information of master variables for the instrument itself, and increasing the data size of the data stream to form the multiple services and a huge data foundation that support big data and industrial interconnection.

Moreover, this involves breakthroughs in the technologies related to the network equipment that are compatible with a variety of wired and wireless communications and integrating a variety of communication modes from the data link to improve the networking capacity of the intelligent instrumentation and the richness of the communication network nodes. It also involves constructing the industrial Internet of Things to get through the last mile of the instrumentation sensor network and promoting the development of higher-end intelligent services and functions of the instrumentation along with highlighting the value of the industrial interconnection data of the instrumentation.

\subsection{Field of medical instruments}

This involves promoting the development of science and technology and the disruptive technologies of medical products.
In the field of medical imaging, the focus is on the development and application of molecular imaging, brain magnetic imaging, fluorescence imaging, terahertz magnetic imaging, electrical impedance imaging, laser imaging, virtual imaging, and other future new technology products. With respect to first-aid field, the focus is on the development of self-adaptive breathing machine, intelligent central monitoring, and remote monitoring. In the field of surgery and recovery, the focus is on the development of the intelligent positioning and navigation robots for orthopedic and brain surgeries, intelligent nursing robots, perceptive intelligent artificial limbs, and aid equipment. In the field of clinical examination, the focus is on (i) developing third-generation sequencing technology and novel test technologies; (ii) establishing a personal health management information system based on gene detection; establishing a human genome database; (iii) developing self-developed gene data access and analysis processing software system; constructing a body area network based on human characteristic parameters; (iv) developing a wearable inspection and monitoring equipment of identification parameters for human health signs based on large-scale cloud computing artificial intelligence method; and (v) researching the application of the resources of the gene pool. In the field of active implants, the focus is on the development of the wireless pacemakers, nerve stimulators, and implanted biochips for treatment and monitoring with better electromagnetic compatibility.

Correspondingly, this is characterized by the rapid development of platform technologies including medical experience mining technology, intelligent medical technology, medicaloriented intelligent technology, cloud diagnostic technology, intelligent operation platform technology of medical resources, and expert systems and medical path description systems.

\subsection{Field of MEMS}

Further research and focus is required to make a breakthrough in MEMS-based integration design and simulation technology, such as MEMS manufacturing technology of advanced functional materials, chip-level MEMS integration technology, MEMS reliability technology, and MEMS testing and standardization technology among others. It also involves the development of various types of domestic micro-nano manufacturing and testing equipment.

This prioritizes designing research directions for the development strategy of instrumentation engineering science and technology in 2035 including high-reliability system-level MEMS forward design theory, complex 3D MEMS manufacturing process method, monolithic MEMS integration method, MEMS application method under extreme conditions, MEMS integration RF front-end technology, micro-navigation positioning and timing technology, MEMS intelligent sensing micro-system technology, and MEMS actuator technology. 


\section{Basic research directions}

\subsection{Field of intelligent instrumentation}

This involves examining (i) the security technology of sensor level-related equipment, link, data security processing and function; (ii) intelligent instrumentation that is adapted to full life-cycle data management; (iii) local and remote selflearning adjustment functions with instruments adapted to working conditions; (iv) local and remote data redundancy functions of instrumentation; remote upgrade and backtracking functions of instruments; automatic preventive detection and maintenance functions of the instrument; and (v) high-precision measurement method based on artificial intelligence and improving ontology measurement capacity and measurement precision of various types of instruments, and the ontology hardware and software diagnostic techniques of instruments [13].

It also involves studying construction technology of instrument networking, communication technology of the transmission layer, full life-cycle data transmission means that can support the integration of service data and control data of the instrumentation and focusing on examining the new industrial high-speed two-wire communication network and network communication card, communication chip, and communication security that supports big stream data transmission. Additionally, this involves investigating core technologies of the equipment and communication of the convergence layer and technologies related to key network equipment, network interface card, and network security that support multi-link diagnosis and analysis and aggregation and separation of service and control data with different characteristics. Furthermore, master station data processing, route processing and flow control, and data security and priority communication scheduling technologies that are adapted to a traditional fieldbus are examined. Moreover, communication scheduling and transmission technologies of the backbone layer, and data exchange and distributing equipment that support the control network and serve internet separation and research data separation and other network security technologies are studied in addition to, (i) safety communication technology based on the industrial Internet of Things and security transmission and, (ii) redundancy of the intelligent manufacturing system based on the industrial Internet of Things.

\subsection{Field of medical instruments}

This focuses on systematic research on the new imaging mechanism of medical imaging, image fusion technology, and remote image diagnosis information along with research on new surgical instruments and instrument and equipment, new first-aid equipment engineering supporting technology with the application of the new technology and basic theory and engineering technology of the new intelligent first-aid instrument and equip- ment that is performed based on the characteristics of clinical diseases. It involves implementing the application of instrument and equipment mechanism and engineering technology for advanced therapies. It also involves implementing the application of the biomechanical basis of intelligent medical robots, and performing research on soft-bodied robot technology, remote surgery technology, medical big data analysis technology based on artificial intelligence, assisted interventional therapy technology, and intelligent technology and biomechanical basis of bionic limbs and aids. Additionally, this develops biochips that can predict the risk of major diseases and develop a complete genome sequencing technology and the full-line, high-throughout, integrated, and procedural laboratory inspection and analysis system. Furthermore, the gene access and analysis system, and basic research on the application of the gene resources, are developed. This focuses on promoting the development of the intelligent memory orthopedic implants, intravascular stents, and applied biological materials with better biocompatibility and examining new intelligent biological materials. It also involves increased efforts to examine the applications of the properties of the biological materials and processing technology and disposal technology, the basis of biocompatibility and biomechanical, and the basis of active implants and biological fusion [13].

\subsection{Field of MEMS}

The focus involves making a breakthrough in cutting-edge science research in the field of MEMS. Specifically, MEMS manufacturing and the forward design of integration between a device and a microsystem are of crucial importance. This involves breakthroughs in forward design theory and methods, and giving priority to studies examining the modeling and simulation of a cross-scale multi-domain systems, characterization of material properties and process compatibility, failure mechanisms and verification methods under a harsh environment to solve problems in MEMS device modeling and simulation, and a forward design theory and method.

Core technologies and processes in the field of MEMS are examined. These include investigating monolithic integration, $3 \mathrm{D}$ integration and mixed integration methods, the advanced structure and material technologies, and the wafer-level package and online monitoring of MEMS to solve the chip-level integration method and the core key process and technology of the MEMS device and micro-system.

Breakthroughs in key applications in the field of MEMS are important. They emphasize the adaptability of the application environment of MEMS devices, as well as microsystems and systems including MEMS devices and systems for engines and gas turbines, ultra-precision and high overloaded MEMS devices and systems for tactical weapons, high-reliability and high-performance MEMS devices and systems for the automobile industry, and MEMS devices and systems for the integration 
and intelligentization of the future Internet of Things to solve the major strategic demand problems in China, which are in urgent need of breakthroughs.

\section{Conclusions}

The continued improvements in MEMS technology will lead to an increase in its application. Micromation is an inevitable development trend of future instrumentation. As a result, micro-instrumentation, in addition to being used in traditional instrumentation, will also play a unique role in automation, aerospace engineering, military affairs, biology, medical treatment, and other fields.

Multi-functionalization is an important trend in instrumentation, and multifunctional integrated products provide a better solution to a variety of test functions.

Owing to the rapid development of network technology, the Internet of Things is gradually infiltrating the design field of industrial control and intelligent instrumentation systems. Additionally, the concept of networked instrumentation signifies a breakthrough in the concept of traditional measuring instruments.

It is expected that artificial intelligence technology will be more widely used in the field of instrumentation and that computers can be used to simulate artificial intelligence. Instrumentation will be further intelligentized in the future, and this can be used to replace a part of the workings of the human brain. Furthermore, instrumentation also shares certain subhuman capacity in terms of vision, hearing, thinking, and other aspects. The application of artificial intelligence in the field of instrumentation is expected to solve problems that cannot be fundamentally solved with traditional methods and to significantly improve production efficiency and capacity.

\section{References}

[1] Ministry of Industry and Information Technology of the People's
Republic of China. Guidelines, goals, highlights and methods for development and structure adjustment of manufacturing industry in "12th Five-Year Plan" [R]. Beijing: Ministry of Industry and Information Technology of the People's Republic of China, 2010. Chinese.

[2] Instrumentation Technology and Economy Institute. Market research report on sensor and instrumentation technology [R]. Beijing: Instrumentation Technology and Economy Institute, 2012. Chinese.

[3] Zhuang S L, Xiao Z H. Development of science and technology in instrumentation, measurement and control area of China $[\mathrm{J}]$. Process Automation Instrumentation, 2009, 30 (5): 1-6. Chinese.

[4] Ministry of Industry and Information Technology of the People's Republic of China. Plan for accelerating the development of sensor and intelligent instrumentation industry [R]. Beijing: Ministry of Industry and Information Technology of the People's Republic of China, 2013. Chinese.

[5] Cabinet Meeting of Japan. Science and technology basic policy [R]. Tokyo: Cabinet Meeting of Japan, 2006.

[6] Chinese Academy of Engineering. Research on the manufacturing power strategy $[\mathrm{M}]$. Beijing: Electronic Industrial Publishing House, 2015. Chinese.

[7] Office of science and technology policy [EB/OL]. (2016-05-10) [2016-10-25]. www.whitehouse.gov/administration/eop/ostp.

[8] Zhao Y H. A Study on development, challenges and strategies of instrumentation industry in China [J] China Instrumentation, 2009 (3): 29-33. Chinese.

[9] Chen Q. A brief probe into trend and application foresight of intelligent instruments $[\mathrm{J}]$. Science and Technology Innovation Herald, 2009 (6): 16. Chinese.

[10] Sun B L. From 2014 new trends see 2015 years instrument industry development direction [J]. Electronic Instrumentation Customer, 2015 (1): 5-10. Chinese.

[11] Yuan T. Summary of the development of the instrument and meter industry in China [J]. Electronic Test, 2016 (6): 122-123. Chinese.

[12] Song Y H. Development of intelligent instrumentation in China [J]. Science and Technology Innovation Herald, 2013 (25): 233.

[13] Xie S F, Wang T, Wang S. Considerations on the healthy development of China's instrumentation industry [R]. Beijing: China Instrument and Control Society, 2010. Chinese. 